\title{
Aplicação de medicamentos por via intramuscular: análise do conhecimento entre profissionais de enfermagem
}

\author{
INTRAMUSCULAR MEDICATIONAPPLICATION: \\ ANALYSIS OF KNOWLEDGE AMONG NURSING PROFESSIONALS
}

APLICACIÓN DE REMEDIOS POR VÍA INTRAMUSCULAR:

ANÁLISIS DEL CONOCIMIENTO ENTRE PROFESIONALES DE ENFERMERÍA

\section{Simone de Godoy ${ }^{1}$, Maria Suely Nogueira ${ }^{2}$, Isabel Amélia Costa Mendes ${ }^{3}$}

\begin{abstract}
RESUMO
Utilizando-se questionário contendo questões abertas e fechadas, o presente estudo teve como objetivos: identificar entre profissionais de enfermagem quais as regiões mais utilizadas para aplicação de medicamentos intramuscular; identificar o método que utilizam para delimitação das regiões e local de punção, identificar seu conhecimento acerca das complicações e contra-indicações para uso das regiões e averiguar a utilização da região ventro-glútea.

Participaram do estudo 32 profissionais que referiram utilizar as regiões dorsoglútea (65,62\%) e deltóidea

(31,25\%). Evidenciou-se que há necessidade de atualização desses profissionais, especialmente quanto a anatomia, utilização adequada de terminologia para denominação das regiões e conhecimentos acerca de complicações e contra-indicações. É essencial o investimento em treinamento desses profissionais para a utilização da região ventroglútea.
\end{abstract}

\section{PALAVRAS-CHAVE}

Injeções intramusculares. Equipe de enfermagem.

\author{
ABSTRACT \\ Using a questionnaire with \\ open and closed questions, this \\ study aimed to: verify among \\ nursing professionals which \\ are the most used regions for \\ intramuscular medication \\ application; identify the \\ method they use for delimiting \\ the puncture regions and site; \\ identify their knowledge of \\ complications and contra- \\ indications of using the \\ regions and verifying the use \\ of the ventrogluteal region. \\ Thirty-two professionals \\ participated in the study, which \\ used the dorsogluteal \\ (65.62\%) and deltoid \\ (31.25\%) regions. The need \\ for updating these \\ professionals was disclosed, \\ especially regarding to \\ anatomy, the adequate \\ terminology used for \\ designating the regions and \\ the knowledge of \\ complications and \\ contraindications. Investments \\ in training these professionals \\ are essential for using the \\ ventrogluteal region.
}

\section{KEYWORDS}

Injections, intramuscular. Nursing staff.

\begin{abstract}
RESUMEN
Por medio de un cuestionario con preguntas abiertas y cerradas, los objetivos del presente estudiofueron: averiguar cuáles son las regiones más utilizadas para la aplicación de remedios por vía intramuscular entre profesionales de enfermería; identificar el método que utilizan para la delimitación de las regiones y el sitio de punción; identificar su conocimiento a cerca de las complicaciones y contraindicaciones en el uso de las regiones y verificar la utilización de la región ventroglutea. Participaron del estudio 32 profesionales que utilizan las regiones dorsoglutea $(65,62 \%)$ y deltoidea $(31,25 \%)$. Se demostró que existe necesidad de actualización de esos profesionales, especialmente con respecto a la anatomía, utilización adecuada de la terminología para la denominación de las regiones y conocimientos a cerca de complicaciones y contraindicaciones. Es esencial la inversión en capacitación de esos profesionales para la utilización de la región ventroglutea.
\end{abstract}

\section{PALABRAS CLAVE}

Inyecciones intramusculares. Grupo de enfermería.

\author{
1 Enfermeira. Especialista \\ em Laboratório do \\ Centro de Comuni- \\ cação \& Enfermagem. \\ Departamento de \\ Enfermagem Geral e \\ Especializada da \\ Escola de Enferma- \\ gem de Ribeirão Preto \\ - USP (EERP/USP). \\ Centro Colaborador \\ da OMS para o \\ desenvolvimento \\ da Pesquisa em \\ Enfermagem. \\ sig@eerp.usp.br \\ 2 Enfermeira. Professor \\ Associado do \\ Departamento de \\ Enfermagem Geral e \\ Especializada da \\ EERP/USP. Centro \\ Colaborador da OMS \\ para o desenvolvimento \\ da Pesquisa em \\ Enfermagem. \\ msnog@eerp.usp.br \\ 3 Enfermeira. Professor \\ Titular do Departamento \\ de Enfermagem Geral \\ e Especializada da \\ EERP/USP. Centro \\ Colaborador da OMS \\ para o desenvolvimento \\ da Pesquisa em \\ Enfermagem. \\ iamendes@eerp.usp.br
}


(a) Considerou-se neste estudo profissionais de enfermagem as categorias de enfermeiro e auxiliar de enfermagem.

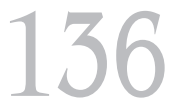

Rev Esc Enferm USP 2004; 38(2): 135-42.

\section{INTRODUÇÃO}

A administração de um medicamento por via intramuscular envolve mais do que a injeção de uma solução no interior da massa muscular, mas também uma avaliação sobre a melhor região e músculo a ser selecionado. Tal procedimento deve ser realizado por pessoas que possuam conhecimentos sobre os aspectos fundamentais de sua execução, ou seja, a equipe de enfermagem. Embora possa ser considerado por muitos como um procedimento relativamente simples, a prática profissional e a literatura consultada apresentam vários relatos de complicações relacionadas à aplicação de medicamentos por esta via.

$\mathrm{Na}$ literatura, foram encontrados relatos de lesões de necrose tecidual, contratura de grupos musculares, fibrose e até perda de amplitude de movimentos articulares em crianças e adultos que fizeram uso de medicação intramuscular ${ }^{(1-6)}$. É importante ressaltar que grande parte dessas complicações ocorre principalmente quando se utilizam os músculos deltóide, glúteo máximo e vasto lateral da coxa, que podem ser traduzidos como os eleitos para aplicação de medicações intramuscular na prática de enfermagem.

Não foram encontrados relatos de complicações devidas à aplicação de medicamentos por via intramuscular nos músculos glúteos médio e mínimo, utilizados quando é selecionada a região ventroglútea. Na busca de alternativas para eliminar as complicações relacionadas ao uso do músculo glúteo máximo, em 1954, o anatomista suíço Von Hochstetter identificou e comprovou, por meio de várias investigações, que a região ventroglútea é o local que menor risco oferece ao cliente, devido ao fato de ser livre de vasos ou nervos importantes e à menor espessura de seu tecido subcutâneo, se comparado aos músculos das regiões dorsoglútea, face ântero-lateral da coxa e deltóidea, também utilizados para a administração de medicamentos por via intramuscular ${ }^{(7)}$.

Tanto as complicações descritas na literatura referentes à utilização das regiões deltóidea, dorsoglútea e face ântero-lateral da coxa, como também a observância da prática revelam baixa adesão na escolha da região ventroglútea. Dessa forma, entende-se que a mudança dessa realidade depende da equipe de enfermagem, que recebendo treinamento adequado e sendo supervisionada talvez passe a incorporá-la em sua prática. Cabe à equi- pe de enfermagem, durante o tratamento de um cliente que necessita de um medicamento intramuscular, assegurar que o procedimento seja feito da maneira mais segura possível, evitando assim complicações relacionadas à aplicação de medicamentos por essa via.

Além do estudo de Castellanos em 1977, não foram identificadas outras investigações sobre o conhecimento da equipe de enfermagem acerca das regiões indicadas para aplicação de medicamentos por via intramuscular. Foram consultadas as bases de dados LILACS, MEDLINE e BEDENF. O fato de não terem sido encontrados relatos de complicações decorrentes da utilização da região ventroglútea motivou o desenvolvimento do presente estudo, que teve como objetivos:

1. identificar quais as regiões mais citadas para aplicação de medicamentos por via intramuscular por profissionais de enfermagem $^{\text {(a) }}$ das unidades de internação cirúrgica e ginecológica de um HospitalEscola do interior paulista;

2. identificar o método que utilizam para delimitar as quatro regiões indicadas para aplicação de medicamentos por via intramuscular e o local de punção;

3. identificar o conhecimento que possuem acerca das complicações e contra-indicações para o uso das regiões utilizadas para aplicação de medicamentos intramuscular e

4. averiguar se a região ventroglútea é utilizada para esse fim.

\section{METODOLOGIA}

A proposta do estudo foi apresentada à chefia da Divisão de Enfermagem de um Hospital Escola do interior paulista, solicitando autorização para seu desenvolvimento. Foi encaminhada ao Comitê de Ética em Pesquisa (CEP) da instituição, e uma vez aprovada, foram feitos contatos com as diretoras de serviço das unidades de internação cirúrgica e ginecológica para a identificação do número de profissionais da equipe de enfermagem. Por meio das escalas mensais, foram identificados 144 profissionais, sendo que $38(26,38 \%)$ estavam em férias, afastados ou de folga por período igual ou superior a três dias, na data selecionada para a coleta de dados, que compreendeu os meses de julho e agosto de 2001. Passou-se a contar com 106 profissionais. 
Utilizou-se para a coleta de dados um questionário contendo questões abertas e fechadas. O planejamento inicial para a coleta de dados previa a entrega dos questionários no início de cada turno, solicitando que fossem devolvidos ao final deste. Considerando a dificuldade relatada pela maioria dos profissionais em devolver o questionário respondido ao final do turno de trabalho, optou-se por permitir sua devolução no turno subseqüente àquele em que o sujeito da pesquisa estivesse escalado para o trabalho.

De 106 profissionais, 12 (11,32\%) não aceitaram o convite para participar do estudo. Foram entregues 94 questionários, 46 $(48,93 \%)$ não foram devolvidos, $03(3,19 \%)$ devolvidos em branco e $33(35,10 \%)$ devolvidos respondidos. Entre estes, um profissional não respondeu as questões referentes à identificação e foi excluído. Desse modo, a amostra do presente estudo foi constituída por 32 sujeitos, os quais assinaram o termo de consentimento livre e esclarecido. A análise dos dados obtidos nos questionários foi efetuada com base na literatura pertinente ao tema ${ }^{(7-9)}$.

Para a descrição do método de delimitação das regiões, foi feito um julgamento das respostas que foram classificadas como correta ou incorreta, sendo consideradas corretas as respostas que contemplaram a literatura em relação à delimitação anatômica, bem como a terminologia utilizada. Foram consideradas incorretas aquelas que, além de confusas, não mencionaram referências anatômicas imprescindíveis para uma delimitação segura.

\section{RESULTADOS E DISCUSSÃO}

Dos 32 profissionais que participaram do estudo, 27 (84,37\%) eram do sexo feminino e $5(15,62 \%)$ do sexo masculino. A média de idade foi de 34 anos. Com relação à função, $7(21,87 \%)$ eram enfermeiros e $25(78,13 \%)$ auxiliares de enfermagem. A média de tempo de atividade na função atual foi de 6 anos e meio. A média de tempo de trabalho na instituição foi de 5 anos e meio.

Quando questionados sobre a execução da aplicação de medicamentos pela via intramuscular, 14 (43,74\%) profissionais indicaram que às vezes executam o procedimento; $17(53,13 \%)$ referiram executar o procedimento freqüentemente e $1(3,13 \%)$, não executá-lo.
Na Tabela 1, são observadas as respostas dos profissionais quando inquiridos sobre as regiões que podem ser utilizadas para a aplicação de medicamentos por via intramuscular, bem como os músculos correspondentes a cada região.

A região deltóidea foi denominada por 14 $(43,75 \%)$ profissionais de "braço" e 29 $(90,62 \%)$ correlacionaram-na ao músculo deltóide.A região dorsoglútea, foi designada glútea por $15(46,87 \%)$ e por $6(18,75 \%)$ que indicaram o glúteo como o músculo correspondente. A região ventroglútea foi assim denominada por $13(40,63 \%)$ profissionais, correlacionada com os músculos glúteo médio e mínimo por apenas por $2(6,25 \%)$ profissionais. A região da face ântero-lateral da coxa foi denominada por $9(28,12 \%)$ profissionais como "coxa" e $25(78,12 \%)$ relacionaram-na ao músculo vasto lateral da coxa.

A terminologia é parte integrante e essencial do contexto teórico e prático de uma profissão. As palavras empregadas entre os elementos de um grupo profissional devem transmitir a todos a mesma significação, evitando perdas e distorções de informações ${ }^{(10)}$.

Neste estudo, pôde-se identificar que a terminologia utilizada pelos profissionais para designar regiões e músculos correspondentes indicados para a aplicação de medicamentos por via intramuscular, foi diversificada, genérica e imprecisa. Foram utilizados vários termos para descrição das regiões e músculos correspondentes. A região da face ântero lateral da coxa foi denominada de "região da perna", "região dos membros inferiores", "coxa" e "face anterior da coxa"; a região deltóidea como "região dos membros superiores", "braço" e "antebraço". A região ventroglútea foi denominada de "região ilíaca" e sua musculatura identificada como "músculo ponto ventroglúteo", "músculo glúteo-ventral". A musculatura da região dorsoglútea foi denominada como "músculo glúteo-dorsal". Verifica-se que ainda há despreparo destes profissionais de enfermagem no uso da terminologia correta para descrever as regiões utilizadas para a aplicação de medicamentos, bem como para a denominação dos músculos correspondentes, o que evidencia que apesar de terem recebido educação formal, esta não possibilitou sedimentação de conhecimentos que são imprescindíveis para uma assistência precisa e qualificada, bem como seu respectivo registro.
Aplicação de medicamentos por via intramuscular: análise do conhecimento entre profissionais de enfermagem



Rev Esc Enferm USP 2004; 38(2):135-42. 
Simone de Godoy Maria Suely Nogueira Isabel Amélia Costa Mendes

Tabela 1 - Regiões utilizadas para aplicação intramuscular de medicamentos e músculos correspondentes, segundo referências dos profissionais $(n=32)$ (Ribeirão Preto, 2001)

\begin{tabular}{|c|c|c|c|c|c|}
\hline Região anatômica & $\mathbf{n}^{\circ}$ & $\%$ & Músculo correspondente & $\mathbf{n}^{\circ}$ & $\%$ \\
\hline Braço & 14 & 43,75 & Deltóide & 29 & 90,62 \\
\hline Deltóide & 12 & 37,5 & Em branco & 2 & 6,25 \\
\hline Descreveu local & 3 & 9,38 & Braço & 1 & 3,13 \\
\hline Antebraço & 1 & 3,13 & & & \\
\hline Membros superiores & 1 & 3,13 & & & \\
\hline Em branco & 1 & 3,13 & & & \\
\hline Glútea & 15 & 46,87 & Glúteo & 20 & 62,50 \\
\hline Nádega & 10 & 31,25 & Glúteo máximo & 6 & 18,75 \\
\hline Dorsoglútea & 3 & 9,38 & Em branco & 2 & 6,25 \\
\hline Descreveu local & 3 & 9,38 & Glúteo dorsal & 1 & 3,13 \\
\hline \multirow[t]{3}{*}{ Quadrante superior externo } & 1 & 3,13 & Nádegas & 1 & 3,13 \\
\hline & & & Descreveu local & 1 & 3,13 \\
\hline & & & Em branco & 1 & 3,13 \\
\hline Ventroglútea & 13 & 40,63 & Ventroglúteo & 14 & 43,75 \\
\hline Em branco & 10 & 31,25 & Em branco & 12 & 37,50 \\
\hline Descreveu local & 3 & 9,38 & Ponto ventroglúteo & 2 & 6,25 \\
\hline Perna & 2 & 6,25 & Glúteo médio e mínimo & 2 & 6,25 \\
\hline Lateral do quadril & 1 & 3,13 & Glúteo ventral & 1 & 3,13 \\
\hline Ilíaca & 1 & 3,13 & Ponta da bacia & 1 & 3,13 \\
\hline Crista ilíaca & 1 & 3,13 & & & \\
\hline Ponto ventroglúteo & 1 & 3,13 & & & \\
\hline Coxa & 9 & 28,12 & Vasto lateral & 25 & 78,12 \\
\hline Vasto lateral da coxa & 8 & 25,00 & Em branco & 4 & 12,50 \\
\hline Em branco & 5 & 15,62 & Transverso da coxa & 1 & 3,13 \\
\hline Face anterior da coxa & 2 & 6,25 & Grande lateral da coxa & 1 & 3,13 \\
\hline Perna & 2 & 6,25 & Coxa & 1 & 3,13 \\
\hline Face lateral da coxa & 1 & 3,13 & & & \\
\hline Vasto ântero-lateral da coxa & 1 & 3,13 & & & \\
\hline Descreveu o local & 1 & 3,13 & & & \\
\hline Membros inferiores & 1 & 3,13 & & & \\
\hline Terço médio da coxa & 1 & 3,13 & & & \\
\hline $\begin{array}{l}\text { Quadrante superexterno da } \\
\text { coxa }\end{array}$ & 1 & 3,13 & & & \\
\hline
\end{tabular}

Para identificar entre os profissionais qual o local de aplicação que utilizam e como delimitam especificamente cada região, foram apresentadas quatro figuras, correspondendo respectivamente às

regiões ventro-glútea, face ântero-lateral da coxa, dorsoglútea e deltóidea e solicitado aos profissionais que descrevessem a delimitação ao lado das ilustrações (Tabelas 2 e 3).

Tabela 2 - Distribuição dos profissionais de enfermagem quanto à indicação correta e incorreta do local de aplicação (Ribeirão Preto, 2001)

\begin{tabular}{ccccc}
\hline Indicação & $\begin{array}{c}\text { Região } \\
\text { Ventroglútea }\end{array}$ & $\begin{array}{c}\text { Região Face } \\
\text { Antero Lateral } \\
\text { da Coxa }\end{array}$ & $\begin{array}{c}\text { Região } \\
\text { Dorsoglútea }\end{array}$ & $\begin{array}{c}\text { Região } \\
\text { Deltóidea }\end{array}$ \\
\hline Correta & $3(9,38 \%)$ & $11(34,38 \%)$ & $15(46,87 \%)$ & $7(21,88 \%)$ \\
Incorreta & $12(37,50 \%)$ & $5(15,62 \%)$ & $4(12,50 \%)$ & $5(15,62 \%)$ \\
Em branco & $17(53,12 \%)$ & $16(50,00 \%)$ & $13(40,63 \%)$ & $20(62,50 \%)$ \\
\hline
\end{tabular}

Tabela 3 - Distribuição dos profissionais de enfermagem quanto à descrição dos métodos que utilizam para delimitar as regiões apresentadas (Ribeirão Preto, 2001)

\begin{tabular}{lccrr}
\hline Indicação & $\begin{array}{c}\text { Região } \\
\text { Ventroglútea }\end{array}$ & $\begin{array}{c}\text { Região } \\
\text { Face Ântero } \\
\text { Lateral da Coxa }\end{array}$ & $\begin{array}{c}\text { Região } \\
\text { Dorsoglútea }\end{array}$ & $\begin{array}{c}\text { Região } \\
\text { Deltóidea }\end{array}$ \\
\hline Correta & $6(18,75 \%)$ & $3(9,38 \%)$ & $2(6,25 \%)$ & $1(3,13 \%)$ \\
Incorreta & $22(68,75 \%)$ & $25(78,12 \%)$ & $27(84,37 \%)$ & $25(78,12 \%)$ \\
Em branco & $4(12,50 \%)$ & $4(12,50 \%)$ & $3(9,38 \%)$ & $6(18,75 \%)$ \\
\hline
\end{tabular}


Conforme apresentado na Tabela 2, na região ventroglútea 3 profissionais $(9,38 \%)$ indicaram corretamente o local de aplicação; na região da face ântero lateral da coxa, 11 profissionais $(34,38 \%)$ realizaram indicação correta; na região dorsoglútea, 15 profissionais $(46,87 \%)$ o fizeram acertadamente e na região deltóidea, 7 profissionais $(21,88 \%)$ indicaram o local correto. Houve um grande número de respostas deixadas em branco.

No estudo realizado em $1977^{(11)}$ com enfermeiros docentes e assistenciais, sobre as regiões para administração de injeção intramuscular, os profissionais foram solicitados a responder uma questão sobre a localização da punção utilizando esquemas. Obteve-se um alto número de abstenção nas respostas e, em sua revisão bibliográfica, identificou outros estudiosos que apresentaram esquemas solicitando o local exato de punção.

A imprecisão na denominação das regiões, bem como na indicação de seus músculos correspondentes, evidenciada no presente estudo, pode interferir na identificação do local correto de punção. Em concordância com a literatura, reafirma-se a importância destes profissionais conhecerem os pontos anatômicos de referência para a correta localização do local de aplicação da injeção. Além disso, devem estar cientes das contra-indicações de cada local e alertas quanto às possíveis complicações.
A região ventroglútea foi delimitada incorretamente por 22 profissionais $(68,75 \%)$, sendo que chamou atenção o fato de um profissional, que deixou a resposta em branco, ter alegado que "auxiliares de enfermagem não podem administrar intramuscular nessa região", revelando também desconhecimento de seus direitos legais. A face ântero-lateral da coxa foi delimitada incorretamente por 25 profissionais $(78,12 \%)$. A região dorsoglútea, da mesma forma, recebeu delimitação incorreta por 27 profissionais (84,37\%). A região deltóidea foi incorretamente delimitada por 25 profissionais $(78,12 \%)$.

Os dados da Tabela 3 mostraram um elevado número de respostas incorretas no tocante à delimitação das quatro regiões, o que pode indicar o desconhecimento destes profissionais sobre a realização correta do procedimento. Causa preocupação detectar erros na delimitação da região deltóidea, uma vez que, observando a prática, identifica-se que esta ainda é uma das regiões mais utilizadas para aplicações intramuscular. Além disso, a possibilidade da punção inadequada de um vaso é grande nessa região, devido à presença dos vasos circunflexos que podem ser atingidos no caso da localização incorreta do local de aplicação.

Buscando identificar a região mais utilizada pelos profissionais foi solicitado que citassem em ordem de preferência as regiões que utilizam para o procedimento (ver Tabela 4).

Tabela 4 - Distribuição dos profissionais quanto à ordem de preferência para aplicação de medicamentos por via intramuscular (Ribeirão Preto, 2001)

\begin{tabular}{lcrrr}
\hline \multicolumn{1}{c}{ Regiões } & $\mathbf{1}^{\mathbf{a}}$ Opção & $\mathbf{2}^{\mathbf{a}}$ Opção & $\mathbf{3}^{\mathbf{a}}$ Opção & $\mathbf{4}^{\mathbf{a}}$ Opção \\
\hline Ventroglútea & $2(6,25 \%)$ & $2(6,25 \%)$ & $3(9,38 \%)$ & $4(12,50 \%)$ \\
Face antero lateral da coxa & - & $6(18,75 \%)$ & $5(15,62 \%)$ & $3(9,38 \%)$ \\
Dorsoglútea & $21(65,62 \%)$ & $13(40,62 \%)$ & $5(15,62 \%)$ & $1(3,13 \%)$ \\
Deltóidea & $9(28,13 \%)$ & $10(31,25 \%)$ & $9(28,13 \%)$ & $2(6,25 \%)$ \\
Em branco & - & $1(3,13 \%)$ & $10(31,25 \%)$ & $22(68,75 \%)$ \\
\hline
\end{tabular}

A região dorsoglútea foi indicada como a $1^{\mathrm{a}}$ opção por 21 profissionais $(65,62 \%)$ e como segunda opção por $13(40,62 \%)$. A região deltóidea foi indicada como $2^{\mathrm{a}}$ opção por 10 profissionais $(31,25 \%)$ e como $3^{\mathrm{a}}$ e $4^{\mathrm{a}}$ opções. Grande parte das respostas foram deixadas em branco. Verificou-se que as regiões mais utilizadas por este grupo de profissionais são as dorsoglútea e deltóidea.

Esses dados corroboram os resultados de estudo realizado em $1977^{(11)}$, que demonstrou ser a região ventroglútea a menos conhecida, utilizada ou orientada a ser utilizada, tanto por enfermeiros docentes quanto por enfermeiros assistenciais. A autora concluiu que havia um círculo vicioso em que a falta de conhecimentos levava à não utilização dessa região como um dos locais para aplicação de injetáveis por via intramuscular.

Quando inquiridos sobre como tomaram conhecimento dessas regiões para a aplicação de medicamentos intramuscular, 27 pro-
Aplicação de medicamentos por via intramuscular: análise do conhecimento entre profissionais de enfermagem 
Simone de Godoy Maria Suely Nogueira Isabel Amélia Costa Mendes fissionais $(84,37 \%)$ indicaram que foi na escola em que se graduaram, $2(6,25 \%)$ informaram que aprenderam com colegas, $1(3,13 \%)$ que aprendeu em curso de atualização e $2(6,25 \%)$ não responderam. Não houve referência entre estes profissionais indicando aquisição de conhecimento por meio de bibliografia específica. Esses dados revelam que embora o conteúdo relativo ao procedimento tenha sido ensinado, ainda há deficiências nos conhecimentos relacionados aos locais de aplicação de medicamentos intramuscular.

Outro estudo em que dois grupos de alunos foram submetidos a métodos de ensino diferentes para aplicação de injetáveis via intramuscular ${ }^{(12)}$ evidenciou que o grupo que recebeu ensino tradicional obteve desempenho inferior quando comparado ao que utilizou módulos auto-instrucionais, comprovando que esse método de ensino favoreceu melhor assimilação do conteúdo.

Como a maioria dos profissionais informou ter tomado conhecimento das regiões na escola em que se formou, e considerando que nos dias atuais o método de ensino mais utilizado ainda é o tradicional (aula expositiva e demonstração), sugere-se que esforços sejam envidados para que outros métodos de ensino sejam utilizadas, visando melhor aprendizado do aluno.

Quanto a encontrar dificuldades para a realização do procedimento, 25 profissionais $(78,12 \%)$ referiram não tê-las e $7(21,88 \%)$ referiram-nas assim se expressando: "insegurança para o uso da região face ântero-lateral da coxa", "dificuldade com crianças que precisam várias aplicações e possuem massa muscular pequena", "não entender porque a região ventroglútea é melhor, pois é difícil de localizar".

A maioria dos profissionais revelou não encontrar dificuldades na realização do procedimento, porém há uma incoerência com os dados encontrados nas respostas, em que se verificou que esses profissionais têm dificuldades para delimitar corretamente as regiões.

Habilidade, destreza e proficiência num procedimento técnico tal como a injeção intramuscular, são obtidas por prática diligente que, para o alcance de resultados apropriados, deve estar baseada em conhecimento científico $^{(7)}$.
Para uma assistência livre de riscos ao cliente é importante que profissionais que realizam procedimentos invasivos saibam identificar suas contra-indicações e complicações. Questionou-se esse conhecimento e verificou-se que os profissionais reconhecem a existência destas, porém ao relacionarem as contra-indicações e complicações, identificou-se certa confusão em diferenciarem as duas terminologias, fato que ficou evidenciado pelas respostas transcritas a seguir.

$\mathrm{Na}$ descrição das contra-indicações foram utilizadas as seguintes expressões: "Voltaren só no glúteo", "deltóide por ser muito vascularizado", "volume no deltóide até $3 \mathrm{ml}$ ", "uso de ângulos curtos próximo à medula para não lesar nervos", "estar atenta à quantidade e tipo de medicação" e; como complicações: "não aplicar antiinflamatório no deltóide", "edema do deltóide", "aplicar medicações oleosas nas nádegas, pois o músculo é maior", "quando se pega algum osso ou nervo".

Novamente identificou-se que, apesar de terem recebido educação formal, os profissionais participantes do presente estudo demonstraram insegurança e incorreção ao expressarem seus conhecimentos relativos à aplicação de medicamentos por via intramuscular.

Considerando que a terminologia pode expressar o saber da enfermagem em suas atividades práticas, passa a ser necessário que a terminologia técnico-científica seja melhor utilizada para que a comunicação entre os profissionais seja eficaz.

A terminologia deve ter constante ressonância com a prática; assim, a terminologia apropriada de uma área do conhecimento é necessidade indispensável ao desenvolvimento da própria ciência. Para que a comunicação entre os profissionais seja clara e eficaz e todos falem a mesma linguagem, é imprescindível o uso da linguagem científica ou tecnológica, isto é, dos termos próprios de uma área de conhecimento ou campo do saber. O vocabulário técnico-científico preciso e exato é um importante instrumento de pesquisa e dá sustentação ao arcabouço teórico da própria ciência ${ }^{(13)}$.

Além de utilizar terminologia apropriada é importante que os profissionais utilizem os 
mesmos critérios ao selecionar a região mais segura para aplicação de medicamentos por via intramuscular. A análise que subsidia a escolha do local adequado para a administração de injetáveis intramuscular é decisiva para um procedimento seguro. Para a seleção do local, deve-se considerar a distância em relação a vasos e nervos importantes, musculatura suficientemente grande para absorver o medicamento, espessura do tecido adiposo, idade, atividade do cliente e irritabilidade da $\operatorname{droga}^{(14)}$.

Os critérios mais utilizados pelos profissionais do presente estudo foram o desenvolvimento muscular, citado por 21 profissionais $(65,62 \%)$, e a irritabilidade da droga, mencionada por todos. Ficou evidenciado que 25 profissionais $(78,13 \%)$ não consideram como critério para seleção do local a atividade do cliente, 13 profissionais $(40,63 \%)$ a idade e 14 profissionais $(43,75 \%)$ a espessura do tecido adiposo. Sérias conseqüências podem ocorrer quando não se consideram critérios como os citados.

Especificamente sobre a região ventroglútea, ao buscar identificar aspectos relacionados à sua utilização, verificou-se que 20 profissionais $(62,5 \%)$ não a utilizam, entre os quais $11(34,38 \%)$ informaram que viram o procedimento apenas na escola e por isso sentem insegurança, $4(12,5 \%)$ nunca viram sua realização, $2(6,25 \%)$ indicaram sentir insegurança, um $(3,13 \%)$ não especificou o motivo e $1(3,13 \%)$ acredita ser mais doloroso para o cliente. Na população estudada somente 5 profissionais $(15,62 \%)$ indicaram que já executaram o procedimento na região ventroglútea.

A identificação de um alto número de profissionais de enfermagem sem nenhuma experiência na aplicação de medicamentos na região ventroglútea confirma que há necessidade de treinamento para que possam adquirir conhecimento técnico-científico e habilidades que thes permitam propiciar atendimento seguro, evitando assim a exposição do cliente às complicações relacionadas à aplicação de medicamentos por esta via.

Os profissionais foram unânimes ao responder que não conhecem contra-indicações ou complicações para a utilização da região ventroglútea.

\section{CONCLUSÕES}

O presente estudo permitiu identificar que:

1. As regiões mais utilizadas por este grupo de profissionais para aplicação de medicamentos intramuscular são as dorsoglútea e deltóidea, mencionadas por 21 profissionais $(65,62 \%)$ e $10(31,25 \%)$, respectivamente.

2. Os profissionais participantes do presente estudo revelaram falhas de conhecimento na descrição do método que utilizam para delimitar as quatro regiões utilizadas para aplicação de medicamentos intramuscular e insegurança para apontar no esquema o local de punção;

3. Houve imprecisão na descrição e confusão na identificação de complicações e contraindicações por esses profissionais e

4. A região ventroglútea ainda é pouco utilizada.

\section{RECOMENDAÇÕES}

No presente estudo, a maioria dos profissionais revelou lacunas de conhecimento em vários aspectos que envolvem a execução do procedimento de maneira eficaz e segura. Os resultados indicam que há uma grande necessidade de atualização desses profissionais. Sugere-se especialmente: revisão da anatomia, com vistas à utilização da terminologia adequada ao denominar regiões e músculos utilizados para a aplicação de medicamentos; revisão dos critérios que devem ser utilizados para a seleção da região mais segura, revisão de conhecimentos acerca das contraindicações e complicações relacionadas ao procedimento e treinamento quanto a delimitação específica e local de punção em cada região. Acredita-se que somente com a solidificação desses conhecimentos os profissionais podem assegurar aos clientes uma aplicação segura, precisa e livre de complicações relacionadas ao procedimento. Ficou evidenciado que a insegurança e o apego aos locais mais comumente usados no Brasil para aplicações intramuscular, ainda estão presentes na atualidade, corroborando os achados e o alerta feitos há $23 \operatorname{anos}^{(7)}$.

Sugere-se que o ensino do procedimento de administração de medicamentos intramuscular seja revisto para que se quebre o "círculo vicioso" relativo ao conhecimento teórico-prático e da pequena utilização de
Aplicação de medicamentos por via intramuscular: análise do conhecimento entre profissionais de enfermagem 
Simone de Godoy Maria Suely Nogueira Isabel Amélia Costa Mendes determinadas regiões. $\mathrm{O}$ ensino deve proporcionar aos profissionais a oportunidade de superar suas inseguranças e difículdades para que transformem o atendimento rotineiro e mecânico em uma prática precisa baseada em conhecimento científico.

\section{REFERÊNCIAS}

(1) Golcman B, Golcman R, Castro LGM, Mizoguchi M. Necrose tecidual após injeção intramuscular de diclofenaco de sódio: relato de 4 casos. An bras dermatol 1991; 66(2): 65-9.

(2) Giovannetti M, Machado MAC, Borrelli Junior M, Ikejiri CI, Alonso N, Branco PD. Necrose tecidual: efeito colateral do diclofenaco de sódio, relato de casos e discussão da fisiopatologia. Rev Hosp Clín Fac Med São Paulo 1993; 48(1): 39-42.

(3) Bologna V, Foppiano M, Godoy GDG, Lombardo GL, Malgor LA, Ramonda MN, Valsecia ME. Efectos adversos graves por inyección intramuscular de penicilina benzatínica. Temas enferm actual 1997; 5(23): 29-32.

(4) Cunha FM, Mestriner LA. Contratura subclínica do músculo quadríceps: avaliação clínico-epidemiológica de 1060 escolares de Belo Horizonte na faixa etária de sete a treze anos. Rev bras ort 1997; 32(5): 383-90.

(5) Godinho GG, Freitas JMA, Moreira LA, Andrade Filho JS. Contratura em abdução no ombro do adulto, secundária a banda fibrosa do deltóide. Rev bras ort 1998; 33(9): 689-94.

(6) Cassiani SHB, Rangel SM. Complicações locais pós injeções intramusculares em adultos: revisão bibliográfica. Medicina 1999; 32(4): 444-50.

(7) Castellanos BEP. Região ventroglútea: local seguro para aplicação de injeção por via intramuscular. Enferm novas dimens 1977; 3: 289-93.
(8) Atikinson LD, Murray ME. Fundamentos de enfermagem: introdução ao processo de enfermagem. Rio de Janeiro: Guanabara; 1986. Proporcionado um meio ambiente quimicamente seguro: administração de medicamentos; p. 267-89.

(9) Beyea SC, Nicoll LH. Administration of medications via the intramuscular route: an integrative review of the literature and research-based protocol for the procedure. Appl Nurs Res 1995; 8(1): 23-33.

(10) Simões N. Contribuição ao estudo da terminologia básica de Enfermagem no Brasil. Taxionomia e Conceituação. [dissertação] Ribeirão Preto (SP): Escola de Enfermagem de Ribeirão Preto/USP; 1980.

(11) Castellanos BEP. Estudo sobre as regiões para aplicação de injeção por via intramuscular. Rev Esc Enferm USP 1977; 11(3): 261-324.

(12) Nogueira MS. Aplicação de injetáveis via intramuscular: comparação entre dois métodos de ensino.[tese] Ribeirão Preto (SP): Escola de Enfermagem de Ribeirão Preto/ USP; 1995.

(13) Simões N. Da metalinguagem profissional: a elaboração de um vocabulário técnico-científico na área da enfermagem. [tese] Ribeirão Preto (SP): Escola de Enfermagem de Ribeirão Preto/ USP; 1988 .

(14) Horn BJ. Intramuscular injections. Hosp World 1968; 4: 34

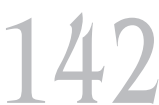

Rev Esc Enferm USP 2004; 38(2): 135-42. 